\title{
Stability of depressive symptoms over 3 months post-partum
}

\begin{abstract}
Aim: Prolonged depression during the post-partum period is associated with maternal and infant mortality and morbidity. Less attention has been given to factors that predict the persistence of depression beyond the first 3 months post-partum.

Methods: From a longitudinal cohort of 2279 women who attended Mazandaran's primary health centres in 2009, 478 women with an Edinburgh Postnatal Depression Scale (EPDS) score of 12 or greater in the third trimester of pregnancy were recruited. Persistently depressed women (depressed at all three occasions: during pregnancy, and at 2 and 12 weeks post-partum) were compared with those without depression to determine demographic, cultural, obstetric and bio psychosocial predictors for persistence of depression. Data were analysed using chi-square test, t-test and logistic regression models.

Results: The stability of depression was found in 193 (46.2\%) of 418 depressed cases who were followed up over the study period. Of those mothers who scored more than the threshold of 12 during the third trimester of pregnancy, 277 (66.3\%) and 221 (52.9\%) had high EPDS at 2 and 12 weeks post-partum. Psychological distress (based upon the General Health Questionnaire), low maternal parental self-efficacy (based upon the Parental Expectation Survey) and perceived social isolation (based upon the Network Orientation Scale) were independent predictors of persistent depression.

Conclusion: Fewer depressed mothers in this study were found to recover during the first 3 months after giving birth. Psychosocial factors predicted sustained depression from pregnancy to 3 months post-partum. The findings highlight the significance of support in enhancing maternal mental health.
\end{abstract}

Keyword: Depression; Post-partum; Risk factor; Stability 